\title{
Mandible reconstruction by the assistant of stereolithographic three-dimensional printing model technique
}

\author{
Cheng-I Yen, MD'; Pang-Yun Chou, MD; Angela Ting-Wei Hsu, MS ${ }^{1,2}$; Mark Shafarenko, BSc ${ }^{3}$; Georgios Kolios, MD, \\ MBA $^{4}$; Chieh-Tsai Wu, MD ; Soo-Ha Kwon, MD ${ }^{1}$; Chien-Tzung Chen, MD ${ }^{6}$; Tommy Nai-Jen Chang, MD ${ }^{1 *}$
${ }^{1}$ Department of Plastic and Reconstructive Surgery, Chang Gung Memorial Hospital, Linkou Medical Center and Chang Gung Medical College and Chang Gung University, Taoyuan, Taiwan
${ }^{2}$ Faculty of Medicine, Mackay Medical College, New Taipei City, Taiwan
${ }^{3}$ University of Toronto, Faculty of Medicine, Toronto, Ontario, Canada
${ }^{4}$ Department of Plastic and Reconstructive Surgery, Agaplesion Diakonie Klinikum, Hamburg, Germany
${ }^{5}$ Department of Neurosurgery, Chang Gung Memorial Hospital, Linkou Medical Center and Chang Gung Medical College and Chang Gung University, Taoyuan, Taiwan
${ }^{6}$ Department of Plastic and Reconstructive Surgery, Chang Gung Memorial Hospital, Keelung Branch and Chang Gung Medical College and Chang Gung University, Taoyuan, Taiwan
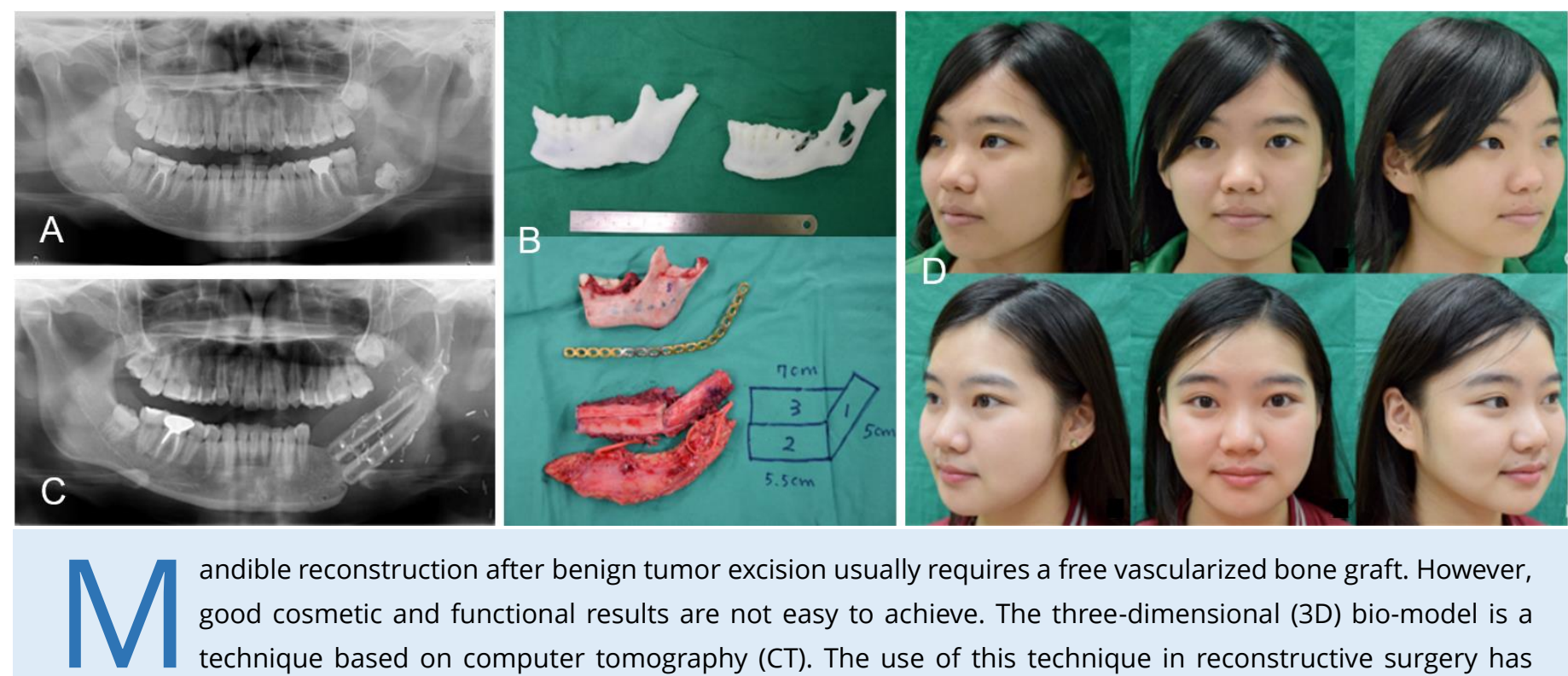

andible reconstruction after benign tumor excision usually requires a free vascularized bone graft. However, good cosmetic and functional results are not easy to achieve. The three-dimensional (3D) bio-model is a technique based on computer tomography (CT). The use of this technique in reconstructive surgery has increased in recent years. The patient suffered from an odontogenic keratocyst (OKC) located between the ramus and angle of the left mandible (Panel A). The 3D stereolithography method was used to design a pre-operative bio-model. One model was designed to define the excision margins of the lesion site. The contralateral site served as a mirror to create the template and a second model was printed to preform the reconstructive plate to the mandible (Panel B upper). After tumor excision, a single stage reconstruction was performed with a left free fibula osteoseptocutaneous flap (Panel B lower). At 18 months follow-up, the bone had good union, and the patient showed a good aesthetic result, proper occlusion, and normal masticatory function (Panel $\mathrm{C}$ and $\mathrm{D}$ ). We propose that the 3D bio-model can assist surgeons to evaluate the size of the defect, design osteotomies tailored to the defect, and shaping of the titanium plate to fit the mandible pre-operatively, which shortens the operative time.

DOI: 10.24983/scitemed.paciv.2017.00009

*Correspondence: Tommy Nai-Jen Chang, MD

Division of Reconstructive Microsurgery, Department of Plastic and Reconstructive

Surgery, Chang-Gung Memorial Hospital; Chang-Gung University, College of

Medicine, Taoyuan, Taiwan. E-mail: tommynjchang@yahoo.com.tw

Received: May 8, 2017; Accepted: May 8, 2017; Published: May 9, 2017

Preprint Archives of Clinical Images \& Videos. 2017;1(2):1

DOI: 10.24983/scitemed.paciv.2017.00009

Copyright $\odot 2017$ The Author(s). This is an open-access article distributed under the terms of the Creative Commons Attribution 4.0 International License (CC-BY). 